\title{
A DELAY FAULT MODEL FOR AT-SPEED FAULT SIMULATION AND TEST GENERATION
}

\author{
Irith Pomeranz \\ School of Electrical \& Computer Eng. \\ Purdue University \\ W. Lafayette, IN 47907 \\ pomeranz@ecn.purdue.edu
}

\author{
Sudhakar M. Reddy \\ Electrical \& Computer Eng. Dept. \\ University of Iowa \\ Iowa City, IA 52242 \\ reddy@engineering.uiowa.edu
}

\begin{abstract}
We describe a transition fault model, which is easy to simulate under test sequences that are applied at-speed, and provides a target for the generation of at-speed test sequences. At-speed test application allows a circuit to be tested under its normal operation conditions. However, fault simulation and test generation for the existing fault models become significantly more complex due to the need to handle faulty signal-transitions that span multiple clock cycles. The proposed fault model alleviates this shortcoming by introducing unspecified values into the faulty circuit when fault effects may occur. Fault detection potentially occurs when an unspecified value reaches a primary output. Due to the uncertainty that an unspecified value propagated to a primary output will be different from the fault free value, an inherent requirement in this model is that a fault would be potentially detected multiple times in order to increase the likelihood of detection. Experimental results demonstrate that the model behaves as expected in terms of fault coverage and numbers of detections of target faults. A variation of an $n$-detection test generation procedure for stuck-at faults is used for generating test sequences under this model.
\end{abstract}

\section{INTRODUCTION}

Application of tests for delay faults in synchronous sequential circuits can be done in one of several ways. Scan can be used to apply two-pattern tests that start and end with scan operations [1]-[3]. Tests can also be applied using only the functional mode of operation of the circuit. In this case it is possible to use slow clock cycles for initialization and fault propagation and fast clock cycles for capturing fault effects [4]-[6]. Alternatively, a test

Permission to make digital or hard copies of all or part of this work for personal or classroom use is granted without fee provided that copies are not made or distributed for profit or commercial advantage and that copies bear this notice and the full citation on the first page. To copy otherwise, to republish, to post on servers or to redistribute to lists, requires prior specific permission and/or a fee.

ICCAD'06, November 5-9, 2006, San Jose, CA

Copyright 2006 ACM 1-59593-389-1/06/0011...\$5.00 sequence can be applied at-speed [7]-[11] using only fast clock cycles. At-speed test application has the advantage that the circuit is tested under its normal operation conditions. It has been shown that certain defects will only be detected if tests are applied at-speed. In addition, as demonstrated in [12], test application that deviates from normal operation can cause faulty behavior that would not show up during normal operation.

Transition faults are used for their simplicity in modeling spot defects that affect delays at inputs or outputs of gates. Under scan based tests transition faults are associated with an extra delay that is large enough to cause the delay of any path through the fault site to exceed the clock period. Beyond this assumption, the specific delay size is not important. When at-speed tests are used, a faulty line is considered under multiple consecutive fast clock cycles. In this case, it becomes necessary to explicitly consider defect sizes measured in numbers of clock cycles in order to determine the value of a faulty line in consecutive fast clock cycles [9]. Thus, it is necessary to consider each transition fault multiple times, associating with it a delay of size $1,2, \cdots$ cycles. This increases the complexity of fault simulation and test generation.

In this work we propose a new delay fault model similar to the transition fault model for use with at-speed tests. The model allows simulation of a given transition fault only once to determine whether it is detected by a given test sequence, and it allows test generation similar to the generation of $n$-detection test sequences for stuckat faults. The model has the following features.

(1) The fault simulation process for the proposed model is similar in complexity to fault simulation of stuck-at faults. (2) The conditions for fault activation are simple to compute. When a fault is activated, the value assigned to the fault site in the faulty circuit is unspecified $(x)$. Unspecified values have several properties that make them suitable for at-speed fault simulation as discussed later.

(3) Each time unit where an unspecified value appears on a primary output is counted as a potential detection of the fault. Due to the uncertainty that the unspecified value will be different from the fault free value, a requirement in this model is that a fault would be detected multiple times. 
(4) The model is shown to be significantly different from the stuck-at fault model by comparing stuck-at faults and transition faults that are related to each other [18]. Experimental results show many cases where a stuck-at fault is detected more times than the related transition fault. The opposite happens in fewer cases. The conclusion is that even $n$-detection test generation for stuck-at faults will not be sufficient for a good coverage of transition faults during at-speed test application.

We assume that at most one transition fault (slowto-rise or slow-to-fall) will occur on each line. The model can be extended to deal with the case where both faults may be present on a line by introducing unspecified values for both types of transitions.

The paper is organized as follows. In Section 2 we introduce the proposed transition fault model. In Section 3 we present the results of fault simulation of test sequences generated for stuck-at faults. The results demonstrate that the model behaves as expected in terms of fault coverage and numbers of detections of target faults. In Section 4 we discuss test generation for the proposed fault model. We use a variation of $n$-detection test generation for stuck-at faults that takes into account the need to increase the numbers of detections of stuck-at faults that are related to undetected transition faults.

\section{DELAY FAULT MODEL}

In this section we describe the proposed delay fault model. The model is similar to the transition fault model, but it is more suitable for at-speed fault simulation and test generation. We refer to faults of the new fault model as unspecified transition faults.

Similar to standard transition faults, we associate an unspecified transition fault with every line $g$ and signaltransition $v \rightarrow v^{\prime}$, for $v \in\{0,1\}$. The fault associated with line $g$ and signal-transition $v \rightarrow v^{\prime}$ is denoted by $g: v \rightarrow v^{\prime}$. Similar to a standard transition fault, the unspecified transition fault $g: v \rightarrow v^{\prime}$ is activated at time unit $u+1$ if $g=v$ at time unit $u$ and $g=v^{\prime}$ at time unit $u+1$. However, when the fault is activated we set the value of $g$ in the faulty circuit to the unspecified value $x$ instead of setting $g$ to the value $v$. The unspecified value on $g$ can then be propagated to the next time units until it eventually disappears. For every time unit where an unspecified value is propagated to a primary output we say that the fault is detected once. The detection is viewed as a potential detection to accommodate the following effects.

(1) The duration of the fault is unknown and different durations may result in different values. As a result, a fault of a certain duration may be detected while a fault of a different duration may not be detected by a given test sequence [9]-[10].

(2) Unmodeled delay effects may speed up a transition and cause the effects of a transition fault not to appear under certain conditions [13]-[17].

For an unspecified transition fault, the higher the number of potential detections, the more likely it is that a defect associated with the same site will actually be detected. Therefore, fault simulation and test generation procedures must consider the numbers of times faults are detected, similar to $n$-detection fault simulation and test generation procedures.

We illustrate the model and the fault simulation process for it by using the example circuit shown in Figure 1. The input sequence under consideration is 0010001010 100010 . The circuit is assumed to be initialized to state 0 before the application of the input sequence. The fault under consideration is $g: 1 \rightarrow 0$. The values throughout the fault free and faulty circuits are shown in Figure 2. $Y$ and $z$ are combined in Figure 2 since they assume the same values at all the time units under the fault $g: 1 \rightarrow 0$. Values are shown in Figure 2 in the form fault-free/faulty. The following points should be noted.

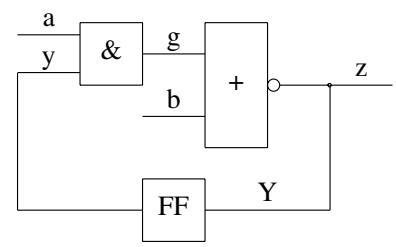

Figure 1: Example circuit
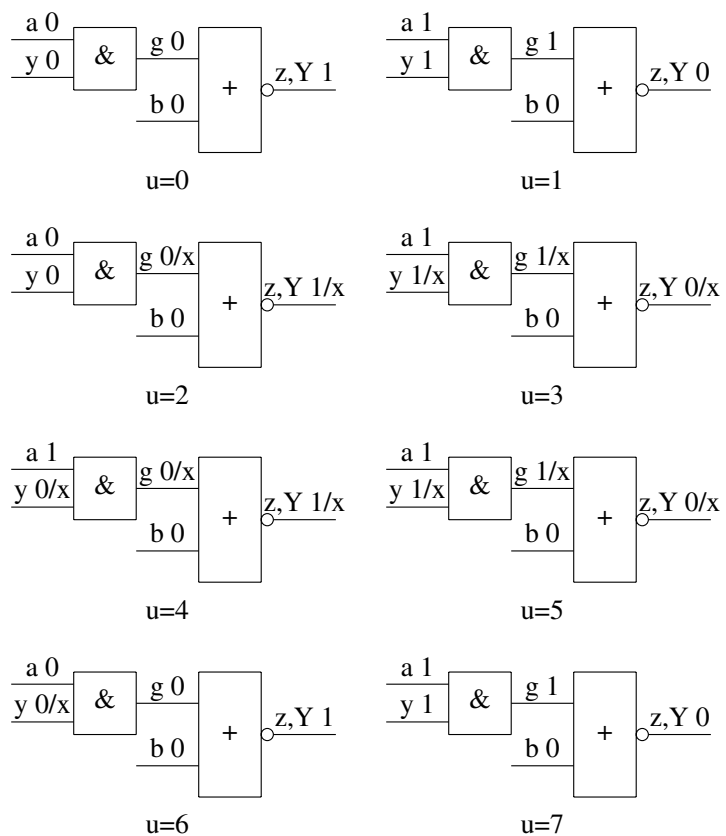

Figure 2: Example circuit with a fault

(1) Since $g=1$ at time unit 1 and $g=0$ at time unit 2, the fault is activated at time unit 2 . This results in the value $g=0 / x$ at time unit 2 .

(2) The fault effect is propagated to the output $z$ at time unit 2. Thus, the fault is detected for the first time. 
(3) At time unit 3, the fault effect continues to propagate. The value $1 / x$ on $g$ is a result of the value $1 / x$ on $y$ at time unit 3. The fault is detected a second time.

(4) If the fault effect had disappeared by time unit 3 and fault free values had been obtained at time units 3 and 4 , the fault would have been injected again at time unit 4 (this would have been based on $g=1$ at time unit 3 and $g=0$ at time unit 4). The unspecified value on $g$ at time unit 4 accommodates this case.

(5) In this example, for every time unit $u$ where the fault is potentially detected since an $x$ is propagated to the output, there is a transition on the output in the fault free circuit. The transition occurs between time units $u-1$ and $u$. In general, an $x$ value can also be propagated to an output at time unit $u$ when the fault free value of the output is the same at time units $u-1$ and $u$.

(6) The values $g=1 / x$ at time unit 5 and $g=0$ at time unit 6 do not cause the fault to be activated again at time unit 6. This is a result of the fact that $g=x$ in the faulty circuit at time unit 5 may not support fault activation at time unit 6.

The importance of point 6 results from the following observations. Unspecified values have the property that injecting a new unspecified value cannot mask an unspecified value injected earlier. A new unspecified value can only increase the likelihood that an unspecified value will be propagated to an output. We introduce an unspecified value into the faulty circuit only when the faulty line has specified values in two consecutive time units. Since the effects of unspecified values add up, by not injecting unspecified values under certain conditions, we may in effect be reducing the number of time units where a fault will be considered potentially detected. As a result, we may be computing a pessimistic estimate of potential fault detections.

The use of unspecified values to mark fault activation and propagation has the following shortcoming. Simulation of unspecified values using three-value logic has an inherent loss of accuracy that may result in an output being unspecified even though more accurate simulation would indicate that the output can only be 0 , or only be 1. However, this effect is small, and it is tolerated in most fault simulation and test generation procedures for synchronous sequential circuits that use three-value logic.

\section{FAULT SIMULATION}

We implemented a fault simulation procedure for the unspecified transition fault model. In this procedure we simulate a fault until it is detected $n$ times, for a constant $n$. For comparison, we also performed $n$-detection fault simulation of stuck-at faults. In this process, a stuck-at fault is considered detected at a time unit $u$ if there exists an output with different specified fault free and faulty values at time unit $u$. A fault is dropped from considera- tion after it is detected at $n$ different time units. For further comparison we also simulated transition faults with an extra delay of a single clock cycle.

The test sequences we simulated are compacted deterministic test sequences generated for stuck-at faults. In every case the circuit is started from the all-zero state. This is done to eliminate unspecified values that occur due to the initial state of the circuit. It is possible to accommodate an unspecified initial state by starting the simulation of unspecified transition faults only after the fault free and faulty circuit states are specified, and ignoring fault activations and fault detections that occur earlier.

The results obtained using $n=5$ are shown in Table 1. Under column flts we show the number of faults (the number of uncollapsed single stuck-at faults, which is equal to the number of transition faults). Under column len we show the length of the test sequence simulated. Under column model we show the fault model being simulated, either single stuck-at faults (row s.a.) or unspecified transition faults (row xtrans). Under column f.c. we show the fault coverage obtained (this is the number of faults detected at least once as a percentage of the total number of faults). Under column ave we show the average number of times a fault is detected by the test sequence. Under column $d=d_{0}$, for $d_{0}=0,1, \cdots, 5$, we show the number of faults detected $d_{0}$ times. Under column 1trans we show the fault coverage of transition faults with an extra delay of a single clock cycle.

From Table 1 it can be seen that the coverage of unspecified transition faults is lower than the coverage of stuck-at faults, and that the numbers of detections of unspecified transition faults are lower as well. The fault coverage is typically somewhat higher than that obtained for transition faults with an extra delay of a single clock cycle. This is consistent with the fact that unspecified transition faults are meant to capture transition faults of different sizes. These results indicate that the unspecified transition fault model behaves as expected, and similar to other delay fault models.

For the purpose of test generation, it is also interesting to see the correlation between the number of detections of an unspecified transition fault $g: v \rightarrow v^{\prime}$ and the number of detections of the related stuck-at fault $g$ stuckat $v$. We say that the faults are related since detection of both faults occurs when the fault changes the value of $g$ from $v^{\prime}$ to a faulty value (a similar relationship exists between standard transition faults and stuck-at faults [18]). For example, we would like to know whether a high (low) number of detections for $g$ stuck-at $v$ implies a high (low) number of detections for $g: v \rightarrow v^{\prime}$. If the correlation between the numbers of detections is high, then test generation for unspecified transition faults can be replaced with $n$-detection test generation for stuck-at faults. 
Table 1: Results of simulation

\begin{tabular}{|c|c|c|c|c|c|c|c|c|c|c|c|c|}
\hline \multirow{4}{*}{$\frac{\text { circuit }}{\text { s208 }}$} & \multirow[b]{2}{*}{ flts } & \multirow[b]{2}{*}{ len } & & \multirow{2}{*}{1 trans } \\
\hline & & & model & f.c. & ave & $\mathrm{d}=0$ & $d=1$ & $\mathrm{~d}=2$ & $\mathrm{~d}=3$ & $\mathrm{~d}=4$ & $d=5$ & \\
\hline & 416 & 105 & s.a. & 70.19 & 2.32 & 124 & 72 & 33 & 36 & 35 & 116 & 51.68 \\
\hline & & & xtrans & 51.68 & 1.47 & 201 & 74 & 41 & 14 & 17 & 69 & \\
\hline s298 & 596 & 117 & & 89.60 & 4.20 & 62 & 10 & 30 & 3 & 29 & 462 & 69.97 \\
\hline & & & xtrans & 69.97 & 3.05 & 179 & 32 & 26 & 21 & 22 & 316 & \\
\hline s344 & 688 & 57 & & 97.38 & 4.69 & 18 & 7 & 12 & 19 & 19 & 613 & 85.47 \\
\hline & & & xtrans & 85.47 & 3.99 & 100 & 18 & 17 & 16 & 39 & 498 & \\
\hline s382 & 764 & 416 & & 96.60 & 4.66 & 26 & 11 & 16 & 12 & 11 & 688 & 73.43 \\
\hline & & & xtrans & 74.08 & 3.18 & 198 & 61 & 30 & 18 & 34 & 423 & \\
\hline s386 & 772 & 121 & & 90.16 & 3.89 & 76 & 55 & 48 & 39 & 34 & 520 & 67.49 \\
\hline & & & xtrans & 67.49 & 2.73 & 251 & 69 & 56 & 16 & 22 & 358 & \\
\hline $\mathrm{s} 400$ & 800 & 611 & & 95.38 & 4.67 & 37 & 7 & 11 & 4 & 13 & 728 & 72.38 \\
\hline & & & xtrans & 72.38 & 3.18 & 221 & 39 & 41 & 23 & 23 & 453 & \\
\hline $\mathrm{s} 420$ & 840 & 108 & & 46.79 & 1.76 & 447 & 71 & 32 & 41 & 26 & 223 & 27.86 \\
\hline & & & xtrans & 27.98 & 0.91 & 605 & 83 & 19 & 8 & 6 & 119 & \\
\hline s510 & 258 & 1020 & & 100.00 & 4.95 & 0 & 3 & 2 & 11 & 9 & 995 & 85.39 \\
\hline & & & xtrans & 85.39 & 4.17 & 149 & 5 & 1 & 29 & 17 & 819 & \\
\hline s526 & 1052 & 1006 & & 86.88 & 4.29 & 138 & 4 & 1 & 9 & 16 & 884 & 59.03 \\
\hline & & & xtrans & 59.13 & 2.71 & 430 & 11 & 51 & 19 & 20 & 521 & \\
\hline s641 & 1280 & 101 & & 88.12 & 4.07 & 152 & 63 & 37 & 30 & 10 & 988 & 75.16 \\
\hline & & & & 75.16 & 3.32 & 318 & 71 & 46 & 55 & 27 & 763 & \\
\hline s820 & 1640 & 491 & & 96.34 & 4.44 & 60 & 67 & 61 & 60 & 52 & 1340 & 74.33 \\
\hline & & & xtrans & 74.51 & 3.43 & 418 & 55 & 50 & 47 & 27 & 1043 & \\
\hline s953 & 267 & 1906 & & 99.37 & 4.56 & 12 & 72 & 92 & 78 & 55 & 1597 & 88.20 \\
\hline & & & xtrans & 88.20 & 3.72 & 225 & 159 & 125 & 120 & 71 & 1206 & \\
\hline s1196 & 2392 & 238 & & 99.87 & 3.82 & 3 & 275 & 368 & 202 & 196 & 1348 & 81.44 \\
\hline & & & xtrans & 81.56 & 2.54 & 441 & 466 & 409 & 225 & 137 & 714 & \\
\hline s1423 & 2846 & 1024 & & 96.94 & 4.34 & 87 & 182 & 137 & 78 & 140 & 2222 & 81.66 \\
\hline & & & xtrans & 83.24 & 4.05 & 477 & 34 & 31 & 39 & 9 & 2256 & \\
\hline s5378 & 10590 & 646 & & 80.34 & 3.81 & 2082 & 291 & 206 & 164 & 101 & 7746 & 71.67 \\
\hline & & & xtrans & 71.72 & 3.37 & 2995 & 286 & 208 & 215 & 105 & 6781 & \\
\hline s35932 & 71864 & 150 & & 89.78 & 4.48 & 7344 & 18 & 43 & 108 & 43 & 64308 & 86.50 \\
\hline & & & xtrans & 86.50 & 4.29 & 9704 & 379 & 183 & 198 & 146 & 61254 & \\
\hline b03 & 768 & 130 & s.a. & 74.22 & 3.42 & 198 & 9 & 35 & 31 & 21 & 474 & 54.17 \\
\hline & & & xtrans & 54.17 & 2.38 & 352 & 10 & 42 & 24 & 42 & 298 & \\
\hline b04 & 2284 & 168 & s.a. & 88.66 & 4.13 & 259 & 76 & 68 & 69 & 49 & 1763 & 71.94 \\
\hline & & & xtrans & 72.42 & 2.66 & 630 & 191 & 279 & 230 & 126 & 828 & \\
\hline b09 & 678 & 269 & s.a. & 84.81 & 3.92 & 103 & 34 & 20 & 9 & 2 & 510 & 64.31 \\
\hline & & & xtrans & 63.72 & 2.92 & 246 & 36 & 5 & 5 & 10 & 376 & \\
\hline b10 & 870 & 190 & s.a. & 93.33 & 4.38 & 58 & 25 & 43 & 10 & 0 & 734 & 70.00 \\
\hline & & & xtrans & 70.57 & 3.21 & 256 & 37 & 35 & 8 & 4 & 530 & \\
\hline b11 & 1830 & 675 & s.a. & 92.40 & 4.57 & 139 & 7 & 13 & 5 & 7 & 1659 & 75.57 \\
\hline & & & xtrans & 75.68 & 3.71 & 445 & 8 & 24 & 5 & 13 & 1335 & \\
\hline
\end{tabular}

In Table 2 we show detailed information about numbers of detections for $s 298$. For every pair $(g, v)$, let $n_{x t r a n s}(g, v)$ be the number of times the unspecified transition fault $g: v \rightarrow v^{\prime}$ is detected, and let $n_{s a}(g, v)$ be the number of times the stuck-at fault $g$ stuck-at $v$ is detected. The pair $(g, v)$ contributes to the entry in row $n_{s a}(g, v)$ and column $n_{\text {xtrans }}(g, v)$ of Table 2 . Thus, the entry in row $i$ and column $j$ of Table 2 provides the number of pairs $(g, v)$ such that $n_{s a}(g, v)=i$ and $n_{x t r a n s}(g, v)=j$.

From Table 2 it can be seen that there are cases where $n_{s a}(g, v)<n$ and $n_{x t r a n s}(g, v)<n$. In these cases, $n$-detection test generation for stuck-at faults may help increase the numbers of detections of unspecified transition faults as well. However, there are also cases where $n_{s a}(g, v)=n$ and $n_{\text {xtrans }}(g, v)<n$. In these cases, it may be necessary to increase the numbers of detections of stuck-at faults that are already detected $n$ times in order to potentially increase the numbers of detections of the related unspecified transition faults. We investigate a variation of an $n$-detection test generation procedure for stuck-at faults as a way to increase the numbers of detections of unspecified transition faults in the next section.
Table 2: Numbers of detections

\begin{tabular}{c|cccccc} 
& \multicolumn{6}{|c}{$n_{\text {xtrans }}$} \\
$n_{s a}$ & 0 & 1 & 2 & 3 & 4 & 5 \\
\hline 0 & 62 & 0 & 0 & 0 & 0 & 0 \\
1 & 2 & 8 & 0 & 0 & 0 & 0 \\
2 & 17 & 2 & 7 & 0 & 1 & 3 \\
3 & 2 & 0 & 0 & 1 & 0 & 0 \\
4 & 11 & 6 & 1 & 9 & 1 & 1 \\
5 & 85 & 16 & 18 & 11 & 20 & 312
\end{tabular}

\section{TEST GENERATION}

In this section we describe a test generation procedure for unspecified transition faults. The procedure attempts to increase the numbers of detections of unspecified transition faults, which are detected fewer than $n$ times by a given test sequence, for a constant $n$.

Based on the discussion of the previous section, we start from a test sequence $T$ for stuck-at faults. We increase the numbers of detections of stuck-at faults by adding test subsequences to $T$ in order to indirectly increase the numbers of detections of unspecified transition faults.

For $d=0,1, \cdots, n-1$, we consider every unspecified transition fault $g: v \rightarrow v^{\prime}$ such that 
$n_{s a}(g, v)>0$ and $n_{x t r a n s}(g, v)=d$. The reason for requiring $n_{s a}(g, v)>0$ is that we will use a subsequence that detects the stuck-at fault $g$ stuck-at $v$ in order to generate a subsequence that potentially detects the related unspecified transition fault $g: v \rightarrow v^{\prime}$.

For every value of $d$ we consider all the faults with $n_{s a}(g, v)>0$ and $n_{\text {xtrans }}(g, v)=d$. For $d>0$ we then consider all the faults with $n_{s a}(g, v)>0$ and $n_{\text {xtrans }}(g, v)=d$ again. This is done in case a fault with $n_{x t r a n s}(g, v)=d-1$ is accidentally detected, and a test subsequences for it may be generated if it is targeted again directly.

When we consider $g: v \rightarrow v^{\prime}$, we obtain a new test subsequence $\hat{T}$ that detects $g$ stuck-at $v$ and concatenate it to $T$. The new test subsequence $\hat{T}$ for $g$ stuck-at $v$ is obtained from $T$ as follows.

We simulate $g$ stuck-at $v$ under the test sequence $T$ starting from the all-unspecified state. If $g$ stuck-at $v$ is not detected by $T$, we do not consider it further. Otherwise, we find the smallest time unit $u_{e}$ where $g$ stuck-at $v$ is detected by $T$. Denoting the subsequence of $T$ that starts at time unit $u_{s}$ and ends at time unit $u_{e}$ by $T\left[u_{s}, u_{e}\right]$, we find in this step a subsequence $T\left[0, u_{e}\right]$ that detects $g$ stuck-at $v$ starting from the all-unspecified state.

We then consider decreasing values of $u_{s}$, $u_{s}=u_{e}, u_{e-1}, \cdots, 0$. We simulate the subsequence $T\left[u_{s}, u_{e}\right]$ of $T$ that starts at time unit $u_{s}$ and ends at time unit $u_{e}$ starting from the all-unspecified state. We stop with the highest value of $u_{s}$ such that $T\left[u_{s}, u_{e}\right]$ detects $g$ stuck-at $v$. Since $T\left[u_{s}, u_{e}\right]$ detects $g$ stuck-at $v$ starting from the all-unspecified state, concatenating $T\left[u_{s}, u_{e}\right]$ to $T$ is guaranteed to increase the number of detections of $g$ stuck-at $v$. To ensure that different test subsequences are obtained when $g$ stuck-at $v$ is considered multiple times, we add the following two steps.

Considering the time units of $T\left[u_{s}, u_{e}\right]$ in a random order, we attempt to omit each test vector from $T\left[u_{s}, u_{e}\right]$. When time unit $u$ is considered, we omit the vector $T[u]$ at time unit $u$ of $T\left[u_{s}, u_{e}\right]$. If $g$ stuck-at $v$ continues to be detected, we accept the omission. Otherwise, we restore $T[u]$ into $T\left[u_{s}, u_{e}\right]$.

After the vector omission step is complete, we randomly decide whether or not to try and change every bit $b$ of $T\left[u_{s}, u_{e}\right]$. When bit $b$ is considered, if the decision is to try and change it, we complement the bit and simulate $g$ stuck-at $v$. If the fault is not detected, we complement the bit again to restore its initial value. Otherwise, we leave the bit complemented.

For illustration we consider the test sequence of $s 27$ shown in Table 3 under column initial. We consider a stuck-at fault $f$ that is detected once by $T$, while the related unspecified transition fault is not detected by $T$. We find that $f$ is detected by $T$ at time unit $u_{e}=8$. Considering $u_{s}=8,7, \cdots$, we find that $T[8,8]=0000$ does not detect $f, T[7,8]=00000000$ does not detect $f$, and so on, until $T[5,8]=1011100100000000$ detects $f$. We set $\hat{T}=T[5,8]=1011100100000000$.

We randomly order the time units of $\hat{T}$ in the order $<1,3,0,2>$. We find that the vector 1001 at time unit 1 of $\hat{T}$ can be omitted, but the remaining vectors cannot be omitted. The resulting test subsequence is $\hat{T}=10110000$ 0000 .

We randomly decide to try and complement bits 0 , 1,2 and 3 at time unit 0 of $\hat{T}$, bit 0 at time unit 1 of $\hat{T}$, and bits 0,1 and 2 at time unit 2 of $\hat{T}$. We find that bits 0,1 and 3 at time unit 0 of $\hat{T}$, bit 0 at time unit 1 of $\hat{T}$, and bits 0 and 2 at time unit 2 of $\hat{T}$ can be complemented. The resulting test subsequence is $\hat{T}=011010001010$. After concatenating $\hat{T}$ to $T$ we obtain the test sequence shown in Table 3 under column extended.

Table 3: Test sequence for $s 27$

\begin{tabular}{r|cc} 
& \multicolumn{2}{|c}{$T[u]$} \\
$u$ & initial & extended \\
\hline 0 & 0111 & 0111 \\
1 & 1001 & 1001 \\
2 & 0111 & 0111 \\
3 & 1001 & 1001 \\
4 & 0100 & 0100 \\
5 & 1011 & 1011 \\
6 & 1001 & 1001 \\
7 & 0000 & 0000 \\
8 & 0000 & 0000 \\
9 & 1011 & 1011 \\
10 & & 0110 \\
11 & & 1000 \\
12 & & 1010
\end{tabular}

The second time the same fault $g$ stuck-at $v$ is considered, the test subsequence $T[5,8]=101110010000$ 0000 is found again. This time, the order of omission is set to $<2,3,0,1>$. Again, only the vector at time unit 1 is omitted to obtain $\hat{T}=101100000000$. We randomly decide to try and complement bits 2 and 3 at time unit 0 , bits 1 and 3 at time unit 1 , and bits 2 and 3 at time unit 2. Bit 3 at time unit 0 , bits 1 and 3 at time unit 1 , and bits 2 and 3 at time unit 2 are complemented without losing the detection of the fault. The resulting test subsequence is $\hat{T}=101001010011$, and it is concatenated to $T$. This test subsequence is different from the one extracted before for the same fault.

We note that even if the same test subsequence $\hat{T}$ is extracted and added to $T$, the state before the application of $\hat{T}$ may be different for the two appearances of $\hat{T}$ in $T$, contributing to different detection conditions of the fault. Experiments reported in [19] indicate that counting detections of a fault as different if they occur in different time units is as effective as using more complex definitions that require stricter conditions.

After concatenating a test subsequence $\hat{T}$ for the fault $g$ stuck-at $v$ to $T$, we simulate the unspecified transition fault $g: v \rightarrow v^{\prime}$. If the number of detections of $g: v \rightarrow v^{\prime}$ does not increase, we remove $\hat{T}$ from $T$ in order not to increase the length of $T$ unnecessarily. 
Results of test generation for the circuits of Table 1 are reported in Table 4. For circuits that are synchronizable using three-value logic, a test subsequence $\hat{T}$ is extracted such that it would detect the target fault starting from the all-unspecified initial state. For other circuits ( $s 510$ and $s 953$ ), a test subsequence is extracted such that it would detect the target fault starting from the final state of the current test sequence $T$.

We show in Table 4 the following parameters before test generation (subcolumn init) and after test generation (subcolumn $t g$ ). The test length is shown under column len. The coverage of stuck-at faults is shown under column f.c. s.a. . The coverage of unspecified transition faults is shown under column f.c. xtrans. The average numbers of detections of stuck-at faults is shown under column ave s.a. . The average numbers of detections of unspecified transition faults is shown under column ave xtrans.

Increases in the stuck-at fault coverage due to test generation are possible in Table 4 since the initial test sequences used were generated assuming an unknown initial state, while we assume that the circuit is initialized to the all-0 state before the test sequence is applied.

In Table 5 we show the numbers of detections of unspecified transition faults before and after test generation. For every $d=d_{0}$, where $d_{0}=0,1, \cdots, 5$, we show the number of unspecified transition faults that are detected $d$ times. The first row for every circuit shows the numbers of detections before test generation, and the second row shows the numbers of detections after test generation.

From Table 4, test generation increases the coverage of detected unspecified transition faults, and their average numbers of detections. From Table 5, relatively few unspecified transition faults remain, that are detected between one and four times.

Table 4: Results of test generation

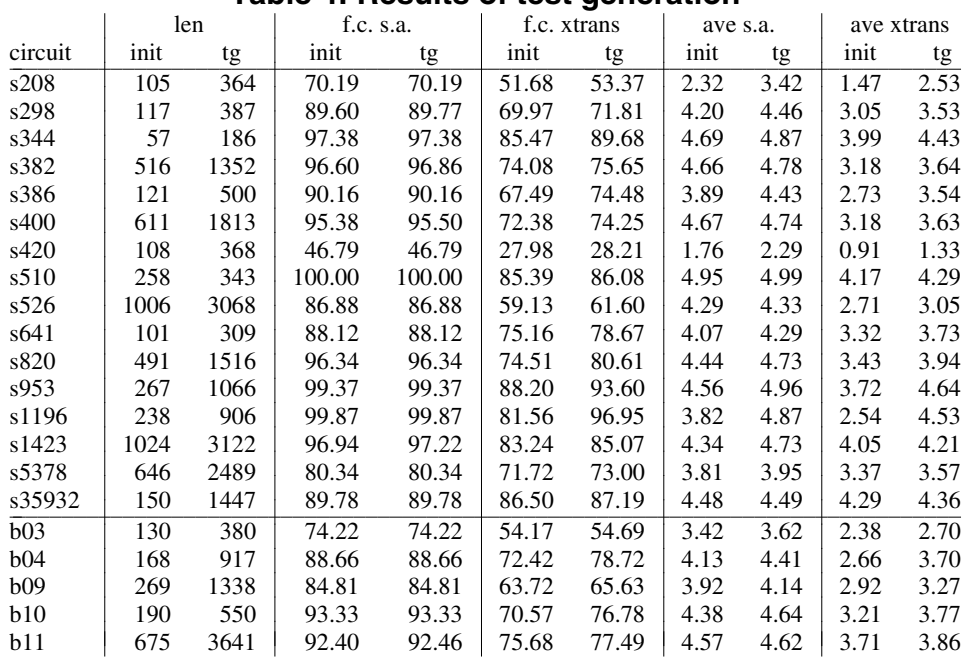

Faults that remain uncovered $(d=0)$ are expected to be undetectable, while faults with five detections may actually have much higher numbers of detections, and defects at these sites are expected to be detected. The small numbers of faults that remain with one to four detections can be targeted directly. The approximately five time increase in test length is consistent with the requirement to detect each fault five times $(n=5)$.

To further demonstrate that $n$-detection test generation for stuck-at faults is not sufficient for ensuring the detection of unspecified transition faults, we compare in Table 6 the numbers of detections of unspecified transition faults before test generation, after $n$-detection test generation for stuck-at faults, and after test generation as proposed here. We consider several circuits for this comparison. The numbers of detections before test generation are shown in row init. The numbers of detections after test generation for stuck-at faults are shown in row $s a$. The numbers of detections after test generation as proposed here are shown in row $x t r$.

Table 5: Numbers of detections after test generation

\begin{tabular}{|c|c|c|c|c|c|c|c|}
\hline circuit & & $\mathrm{d}=0$ & $\mathrm{~d}=1$ & $d=2$ & $d=3$ & $\mathrm{~d}=4$ & $\mathrm{~d}=5$ \\
\hline \multirow[t]{2}{*}{$\overline{\mathrm{s} 208}$} & init & 201 & 74 & 41 & 14 & 17 & 69 \\
\hline & $\operatorname{tg}$ & 194 & 10 & 3 & 4 & 1 & 204 \\
\hline \multirow[t]{2}{*}{$\overline{\mathrm{s} 298}$} & init & 179 & 32 & 26 & 21 & 22 & 316 \\
\hline & $\operatorname{tg}$ & 168 & 5 & 3 & 2 & 2 & 416 \\
\hline \multirow[t]{2}{*}{ s344 } & init & 100 & 18 & 17 & 16 & 39 & 498 \\
\hline & $\operatorname{tg}$ & 71 & 5 & 3 & 0 & 6 & 603 \\
\hline \multirow[t]{2}{*}{ s382 } & init & 198 & 61 & 30 & 18 & 34 & 423 \\
\hline & $\operatorname{tg}$ & 186 & 7 & 23 & 4 & 5 & 539 \\
\hline \multirow[t]{2}{*}{ s386 } & init & 251 & 69 & 56 & 16 & 22 & 358 \\
\hline & $\operatorname{tg}$ & 197 & 19 & 7 & 16 & 11 & 522 \\
\hline \multirow[t]{2}{*}{$\overline{\mathrm{s} 400}$} & init & 221 & 39 & 41 & 23 & 23 & 453 \\
\hline & $\operatorname{tg}$ & 206 & 8 & 9 & 0 & 6 & 571 \\
\hline \multirow[t]{2}{*}{$\mathrm{s} 420$} & init & 605 & 83 & 19 & 8 & 6 & 119 \\
\hline & $\operatorname{tg}$ & 603 & 5 & 11 & 7 & 1 & 213 \\
\hline \multirow[t]{2}{*}{ s510 } & init & 149 & 5 & 1 & 29 & 17 & 819 \\
\hline & $\operatorname{tg}$ & 142 & 2 & 1 & 0 & 0 & 875 \\
\hline \multirow[t]{2}{*}{$\overline{s 526}$} & init & 430 & 11 & 51 & 19 & 20 & 521 \\
\hline & $\operatorname{tg}$ & 404 & 7 & 0 & 2 & 1 & 638 \\
\hline \multirow[t]{2}{*}{ s641 } & init & 318 & 71 & 46 & 55 & 27 & 763 \\
\hline & $\operatorname{tg}$ & 273 & 31 & 25 & 25 & 13 & 913 \\
\hline \multirow[t]{2}{*}{ s820 } & init & 418 & 55 & 50 & 47 & 27 & 1043 \\
\hline & $\operatorname{tg}$ & 318 & 18 & 10 & 14 & 12 & 1268 \\
\hline \multirow[t]{2}{*}{ s953 } & init & 225 & 159 & 125 & 120 & 71 & 1206 \\
\hline & $\operatorname{tg}$ & 122 & 5 & 9 & 9 & 13 & 1748 \\
\hline \multirow[t]{2}{*}{$\overline{s 1196}$} & init & 441 & 466 & 409 & 225 & 137 & 714 \\
\hline & $\operatorname{tg}$ & 73 & 58 & 97 & 71 & 88 & 2005 \\
\hline \multirow[t]{2}{*}{$\overline{\mathrm{s} 1423}$} & init & 477 & 34 & 31 & 39 & 9 & 2256 \\
\hline & $\operatorname{tg}$ & 425 & 11 & 10 & 14 & 8 & 2378 \\
\hline \multirow[t]{2}{*}{ s5378 } & init & 2995 & 286 & 208 & 215 & 105 & 6781 \\
\hline & $\operatorname{tg}$ & 2859 & 114 & 91 & 55 & 44 & 7427 \\
\hline \multirow[t]{2}{*}{$\overline{\mathrm{s} 35932}$} & init & 9704 & 379 & 183 & 198 & 146 & 61254 \\
\hline & $\operatorname{tg}$ & 9205 & 3 & 3 & 1 & 5 & 62647 \\
\hline \multirow[t]{2}{*}{ b03 } & init & 352 & 10 & 42 & 24 & 42 & 298 \\
\hline & $\operatorname{tg}$ & 348 & 0 & 3 & 4 & 8 & 405 \\
\hline \multirow[t]{2}{*}{$\overline{\mathrm{b} 04}$} & init & 630 & 191 & 279 & 230 & 126 & 828 \\
\hline & $\operatorname{tg}$ & 486 & 41 & 57 & 63 & 79 & 1558 \\
\hline \multirow[t]{2}{*}{$\overline{b 09}$} & init & 246 & 36 & 5 & 5 & 10 & 376 \\
\hline & $\operatorname{tg}$ & 233 & 0 & 1 & 1 & 0 & 443 \\
\hline \multirow[t]{2}{*}{$\mathrm{b} 10$} & init & 256 & 37 & 35 & 8 & 4 & 530 \\
\hline & $\operatorname{tg}$ & 202 & 6 & 6 & 7 & 7 & 642 \\
\hline \multirow[t]{2}{*}{$\overline{b 11}$} & init & 445 & 8 & 24 & 5 & 13 & 1335 \\
\hline & $\operatorname{tg}$ & 412 & 1 & 3 & 9 & 0 & 1405 \\
\hline
\end{tabular}


Table 6: Comparison with stuck-at test generation

\begin{tabular}{|c|c|c|c|c|c|c|c|c|c|}
\hline circuit & & len & ave & $\mathrm{d}=0$ & $\mathrm{~d}=1$ & $\mathrm{~d}=2$ & $d=3$ & $\mathrm{~d}=4$ & $d=5$ \\
\hline \multirow[t]{3}{*}{ s298 } & init & 117 & 3.05 & 179 & 32 & 26 & 21 & 22 & 316 \\
\hline & sa & 406 & 3.49 & 168 & 6 & 1 & 12 & 8 & 401 \\
\hline & xtr & 387 & 3.53 & 168 & 5 & 3 & 2 & 2 & 416 \\
\hline \multirow[t]{3}{*}{ s344 } & init & 57 & 3.99 & 100 & 18 & 17 & 16 & 39 & 498 \\
\hline & sa & 116 & 4.38 & 75 & 5 & 4 & 7 & 6 & 591 \\
\hline & xtr & 186 & 4.43 & 71 & 5 & 3 & 0 & 6 & 603 \\
\hline \multirow[t]{3}{*}{ s382 } & init & 516 & 3.18 & 198 & 61 & 30 & 18 & 34 & 423 \\
\hline & $\mathrm{sa}$ & 1219 & 3.58 & 187 & 19 & 12 & 13 & 10 & 523 \\
\hline & xtr & 1352 & 3.64 & 186 & 7 & 23 & 4 & 5 & 539 \\
\hline \multirow[t]{3}{*}{$\mathrm{s} 400$} & init & 611 & 3.18 & 221 & 39 & 41 & 23 & 23 & 453 \\
\hline & sa & 1120 & 3.36 & 211 & 37 & 20 & 22 & 4 & 506 \\
\hline & xtr & 1813 & 3.63 & 206 & 8 & 9 & 0 & 6 & 571 \\
\hline \multirow[t]{3}{*}{$\bar{s} 526$} & init & 1006 & 2.71 & 430 & 11 & 51 & 19 & 20 & 521 \\
\hline & $\mathrm{sa}$ & 2020 & 2.95 & 419 & 2 & 2 & 16 & 17 & 596 \\
\hline & xtr & 3068 & 3.05 & 404 & 7 & 0 & 2 & 1 & 638 \\
\hline \multirow[t]{3}{*}{ s641 } & init & 101 & 3.32 & 318 & 71 & 46 & 55 & 27 & 763 \\
\hline & sa & 370 & 3.86 & 253 & 18 & 15 & 20 & 40 & 934 \\
\hline & xtr & 309 & 3.73 & 273 & 31 & 25 & 25 & 13 & 913 \\
\hline \multirow[t]{3}{*}{ s820 } & init & 491 & 3.43 & 418 & 55 & 50 & 47 & 27 & 1043 \\
\hline & sa & 1832 & 3.89 & 317 & 23 & 17 & 31 & 29 & 1223 \\
\hline & xtr & 1516 & 3.94 & 318 & 18 & 10 & 14 & 12 & 1268 \\
\hline \multirow[t]{3}{*}{ s1196 } & init & 238 & 2.54 & 441 & 466 & 409 & 225 & 137 & 714 \\
\hline & $\mathrm{sa}$ & 1019 & 4.32 & 73 & 79 & 162 & 137 & 177 & 1764 \\
\hline & $\mathrm{xtr}$ & 906 & 4.53 & 73 & 58 & 97 & 71 & 88 & 2005 \\
\hline \multirow[t]{3}{*}{ s1423 } & init & 1024 & 4.05 & 477 & 34 & 31 & 39 & 9 & 2256 \\
\hline & sa & 5394 & 4.23 & 407 & 10 & 22 & 21 & 9 & 2377 \\
\hline & xtr & 3122 & 4.21 & 425 & 11 & 10 & 14 & 8 & 2378 \\
\hline \multirow[t]{3}{*}{$\mathrm{b} 03$} & init & 130 & 2.38 & 352 & 10 & 42 & 24 & 42 & 298 \\
\hline & $\mathrm{sa}$ & 470 & 2.73 & 347 & 0 & 2 & 1 & 4 & 414 \\
\hline & xtr & 380 & 2.70 & 348 & 0 & 3 & 4 & 8 & 405 \\
\hline \multirow[t]{3}{*}{ b04 } & init & 168 & 2.66 & 630 & 191 & 279 & 230 & 126 & 828 \\
\hline & sa & 787 & 3.69 & 488 & 33 & 65 & 50 & 122 & 1526 \\
\hline & $\mathrm{xtr}$ & 917 & 3.70 & 486 & 41 & 57 & 63 & 79 & 1558 \\
\hline \multirow[t]{3}{*}{ b09 } & init & 269 & 2.92 & 246 & 36 & 5 & 5 & 10 & 376 \\
\hline & sa & 1357 & 3.26 & 230 & 3 & 3 & 2 & 3 & 437 \\
\hline & xtr & 1338 & 3.27 & 233 & 0 & 1 & 1 & 0 & 443 \\
\hline \multirow[t]{3}{*}{ b10 } & init & 190 & 3.21 & 256 & 37 & 35 & 8 & 4 & 530 \\
\hline & sa & 463 & 3.64 & 217 & 7 & 17 & 6 & 7 & 616 \\
\hline & xtr & 550 & 3.77 & 202 & 6 & 6 & 7 & 7 & 642 \\
\hline \multirow[t]{3}{*}{ b11 } & init & 675 & 3.71 & 445 & 8 & 24 & 5 & 13 & 1335 \\
\hline & sa & 1556 & 3.83 & 425 & 4 & 0 & 4 & 1 & 1396 \\
\hline & xtr & 3641 & 3.86 & 412 & 1 & 3 & 9 & 0 & 1405 \\
\hline
\end{tabular}

From Table 6 it can be seen that the proposed test generation procedure typically results in higher numbers of detections of unspecified transition faults. The test length is sometimes lower under the proposed procedure than if $n$-detection test generation is carried out for stuck-at faults.

\section{CONCLUDING REMARKS}

We defined a transition fault model for use with at-speed test sequences. The model was referred to as the unspecified transition fault model since it introduces unspecified values into the faulty circuit when fault effects may occur. Fault detection potentially occurs when an unspecified value reaches a primary output. Due to the uncertainty that the unspecified value will be different from the fault free value, a requirement of this model is that a fault would be detected multiple times. Experimental results demonstrated that the model behaves as expected in terms of fault coverage and numbers of detections of target faults. Moreover, an unspecified transition fault may have a significantly smaller number of detections than the related stuck-at fault. Thus, the model provides a target for the generation of at-speed test sequences, which is more effective than $n$-detection test generation for stuck-at faults. A variation of an $n$ detection test generation procedure for stuck-at faults was used for generating test sequences under this model.

\section{ACKNOWLEDGMENTS}

The work was supported in part by SRC Grant No. 2004TJ-1244 (IP) and SRC Grant No. 2004-TJ-1243 (SMR).

\section{REFERENCES}

[1] S. Dasgupta, R. G. Walther, T. W. Williams and E. B. Eichelberger, "An Enhancement to LSSD and Some Applications of LSSD in Reliability, Availability and Serviceability", in Proc. 11th Fault-Tolerant Computing Symp., 1981, pp. 880-885.

[2] J. Savir and S. Patil, "Scan-Based Transition Test", IEEE Trans. on Computer-Aided Design, Aug. 1993, pp. 1232-1241.

[3] J. Savir and S. Patil, "Broad-Side Delay Test", IEEE Trans. on Computer-Aided Design, Aug. 1994, pp. 1057-1064.

[4] S. Devadas, "Delay Test Generation for Synchronous Sequential Circuits", in Proc. Intl. Test Conf., Aug. 1989, pp. 144-152.

[5] T. J. Chakraborty, V. D. Agrawal and M. L. Bushnell, "Delay Fault Models and Test Generation for Random Logic Sequential Circuits", in Proc. Design Autom. Conf., June 1992, pp. 165-172.

[6] I. Pomeranz and S. M. Reddy, "SPADES-ACE: A Simulator for Path Delay Faults in Sequential Circuits with Extensions to Arbitrary Clocking Schemes", IEEE Trans. on Computer-Aided Design, Feb. 1994, pp. 251-263.

[7] I. Pomeranz and S. M. Reddy, "At-Speed Delay Testing of Synchronous Sequential Circuits", in Proc. 29th Design Autom. Conf., June 1992, pp. 177-181.

[8] S. Bose, P. Agrawal and V. D. Agrawal, "Path Delay Fault Simulation of Sequential Circuits", IEEE Trans. on VLSI Systems, Dec. 1993, pp. 453-461.

[9] K.-T. Cheng, "Transition Fault Testing for Sequential Circuits", IEEE Trans. on Computer-Aided Design, Dec. 1993, pp. 19711983.

[10] D. Brand and V. S. Iyengar, "Identification of Redundant Delay Faults", IEEE Trans. on Computer-Aided Design, May 1994, pp. 553-565

[11] W.-C. Lai, A. Krstic and K.-T. Cheng, "On Testing the Path Delay Faults of a Microprocessor Using Its Instruction Set", in Proc. VLSI Test Symp., Apr. 2000, pp. 15-20.

[12] J. Rearick and R. Rodgers, "Calibrating Clock Stretch During AC Scan Testing", Intl. Test Conf., Nov. 2005, pp. 266-273.

[13] P. Franco and E. J. McCluskey "Three-Pattern Tests for Delay Faults", in Proc. 12th VLSI Test Symp., April 1994, pp. 452-456.

[14] A. Pierzynska and S. Pilarski, "Quality Considerations in Delay Fault Testing", in Proc. EURO-DAC 1995, Sept. 1995.

[15] A. Pierzynska and S. Pilarski, "Non-Robust versus Robust", in Proc. 1995 Intl. Test Conf., Oct. 1995, pp. 123-131.

[16] L.-C. Wang, J.-J. Liou and K.-T. Cheng, "Critical Path Selection for Delay Fault Testing Based Upon a Statistical Timing Model", IEEE Trans. on Computer-Aided Design, Nov. 2004, pp. 15501565 .

[17] B. Seshadri, I. Pomeranz, S. M. Reddy and S. Kundu, "PathOriented Transition Fault Test Generation Considering Operating Conditions", in Proc. Europ. Test Symp., May 2005, pp. 54-59.

[18] J. Waicukauski, E. Lindbloom, B. Rosen and V. Iyengar, "Transition Fault Simulation", IEEE Design \& Test, April 1987, pp. 32 38 .

[19] I. Pomeranz and S. M. Reddy, "On $n$-Detection Test Sequences for Synchronous Sequential Circuits", in Proc. 15th VLSI Test Symp., April 1997, pp. 336-342. 